\title{
Editorial
}

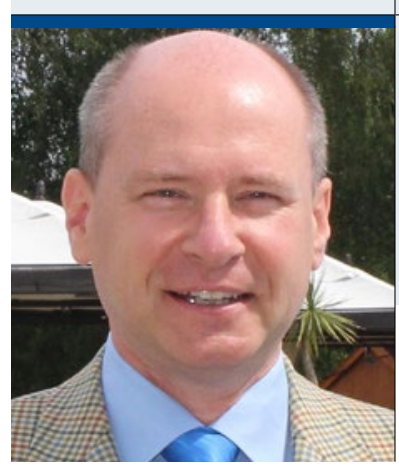

"Erstaunlicherweise hat das wichtige Thema Begutachtung

weder während der akademischen Ausbildung noch während

der fachlichen Weiterbildung einen merklichen Stellenwert."

\section{Der Arzt als medizinischer Sachverständiger und Gutachter}

m Allgemeinen besteht das Selbstverständnis des Arztes in seinem Bemühen um die Behandlung medizinischer Defizite der ihn in der Regel aus freien Stücken konsultierenden Patienten. Dabei übernimmt er - meist unbewusst - die von den $\mathrm{Pa}$ tienten erwartete Rolle als „Anwalt für die Patientengesundheit“, ist also im weitesten Sinne parteiergreifend für und von den Patienten voreingenommen. Grundlage der diagnostisch und therapeutisch gezogenen Konsequenzen sind allein die von dem Patienten vorgetragenen Beschwerden. Demgegenüber bedürfen viele staatliche Behörden und private Institutionen der ärztlichen Hilfe von medizinischen Sachverständigen und Gutachtern, um medizinische Zusammenhänge und Folgen von Unfällen und Erkrankungen sowie deren Auswirkungen in verschiedenen Rechtsbereichen zu beurteilen.

Trotz seiner Aufgabe als Berater und Erfüllungsgehilfe des jeweiligen Auftraggebers, muss sich der Arzt stets seiner Rolle als unparteiische Instanz bewusst sein. Seine Aufgabe ist die objektive Feststellung vorliegender Funktions- und Gesundheitsstörungen und gegebenenfalls der Zusammenhangsbeurteilung mit potenziellen Schädigungsursachen. Im Gegensatz zu dem im Rahmen der allgemeinen Patientenversorgung im Vordergrund stehenden Ziel, zu einer Diagnose und Behandlungsplanung zu gelangen, muss bei einer Begutachtung jedoch immer ein unumstößlicher Vollbeweis angestrebt werden. Dabei kann und wird der zu erbringende und von dem zu Begutachtenden zu ertragende Aufwand mitunter deutlich über dem einer üblichen Patientenversorgung liegen. Auch hier kommt der umfassenden Aufklärung des zu Begutachtenden ein besonderer Stellenwert zu, insbesondere ist er über seine Mitwirkungspflicht sowie zustimmungspflichtige Untersuchungen und Maßnahmen zu informieren.

Ebenso wie der Gutachter lediglich als Berater seines Auftraggebers ohne definitive Entscheidungs- befugnis fungiert, unterliegt er in seiner medizinischen Bewertung und Begutachtung keinerlei Weisungen des Auftraggebers. Die Begutachtung kann jedoch keinesfalls nach Gutdünken des jeweiligen Gutachters vorgenommen werden. Vielmehr hat er sich an die aktuellen medizinisch-wissenschaftlichen Erkenntnisse zu halten, was ein intensives Literaturstudium erforderlich machen kann. Weicht er von allgemeingültigen Erkenntnissen ab, hat er sein Vorgehen plausibel zu begründen. Gerne wird von weniger erfahrenen Gutachtern übersehen, dass für die unterschiedlichen Rechtsbereiche auch unterschiedliche Bewertungsgrundlagen heranzuziehen sind, zum Beispiel für die Beurteilung der Erwerbsfähigkeit. Grundsätzlich sollte sich jeder Gutachter der möglichen Tragweite seiner Bewertungen sowohl für den zu Begutachtenden als auch den Auftraggeber bewusst sein, beispielsweise bei einer monate-, gelegentlich jahrelangen Verzögerung bei der Bearbeitung eines angenommenen Gutachtenauftrags.

Erstaunlicherweise hat das wichtige Thema Begutachtung weder während der akademischen Ausbildung noch während der fachlichen Weiterbildung einen merklichen Stellenwert. Zwar sozialmedizinisch hochrelevant, fristet die Begutachtung aber von jeher bei den meisten Kollegen ein eher ungeliebtes Dasein. Vielleicht ist auch das einer der Gründe für die rar gesäten und auf einige wenige Fachbereiche beschränkten Fort- und Weiterbildungsmöglichkeiten.

Wir möchten in dieser URO-NEWS etwas mehr Verständnis für die Begutachtung schaffen und die wichtigsten Grundlagen vermitteln. In URO-NEWS 2/2018 erfahren Sie dann, wie ein Gutachten, insbesondere der Vollbeweis, speziell bei urologischen Erkrankungen gehandhabt wird.

\section{Dr. med. Michael Zellner}

